\title{
Unified Software Repositories for Wendelstein 7-X: Workflow Elements for Fusion Software Development
}

\author{
A. Kus ${ }^{\mathrm{a}, *}$ A. Krüger ${ }^{\mathrm{b}}$, A. Dinklage ${ }^{\mathrm{a}}$, T. Bluhm ${ }^{\mathrm{a}}$, C.G. Hanke ${ }^{\mathrm{c}}$, G. Kühner ${ }^{\mathrm{a}}$, \\ A. Werner ${ }^{\mathrm{a}}$, Ch. Hennig, ${ }^{\mathrm{a}}$, Geiger ${ }^{\mathrm{a}}$, M. Lewerentz ${ }^{\mathrm{a}}$, Yu. Turkin ${ }^{\mathrm{a}}$, the W7-X team \\ ${ }^{a}$ Max-Planck-Institut für Plasmaphysik, IPP-Euratom Association, Wendelsteinstr.1, 17491 Greifswald, Germany \\ ${ }^{\mathrm{b}}$ University of Applied Sciences, Schwedenschanze 135, 18435 Stralsund, Germany \\ ${ }^{\mathrm{c}}$ Rechenzentrum der Max-Planck-Gesellschaft, Boltzmannstr. 2, 85748 Garching, Germany
}

\begin{abstract}
Software development for a large project like W7X requires an appropriate development process to provide a reliable environment supporting the coordinated work of scientific and engineering stakeholders. The first step to achieve this goal was the installation of a central source code management system (SCM). Besides being a code base it provides functions allowing the specification of a release procedure by introducing levels of acceptance specified by appropriate quality criteria. The SCM system serves as a starting point for automatic generation of standard code documentation and for performing automatic testing procedures on standardized integration platforms. In order to provide a universal documentation platform for the multi programming language environment at W7X the DocSys system has been developed which allows to employ any (language specific) document generator producing html-pages for presentation via internet. An automatic integration testing environment is still being prepared and not yet in standard use.
\end{abstract}

Keywords: Integrated data analysis, Versioning, Automated documentation, Commissioning, Software integration.

\section{Software development process}

Software developments for Wendelstein 7-X aim at an integrated software project [1]. Efforts for device control, data acquisition, data analysis, modelling and theory are to be shared to avoid duplicate work and to gain most validated software modules. Development and implementation of the W7-X software project require a well-designed workflow that allows quality, safety, stability, as well as the necessary flexibility to be adequate for every use-case, ranging from operation relevant software to scientific exploration.

The workflow for W7-X software developments is not finally settled yet. However, central elements are identified and Fig. 1 shows a possible workflow organization. The private/public paradigm is oriented at similar distinctions at JET, where data are organized to be used, e.g., for development (private) or routine (public) purposes. Here, cf. Fig. 1, the developer is working in a private environment first. This can be an

\footnotetext{
* Email address: andreas.kus@ipp.mpg.de
} 
afs directory, a local data medium, or a professional versioning system.

A prerequisite for developments to be released for public use is a commissioning process. Directions for the commissioning process were defined [2] and are going to be extended to be in accordance with respective ISO standards. Up to now, there are development guidelines, specifications of supported programming languages and versioning systems (SVN, CVS) and formal acceptance criteria. A central element

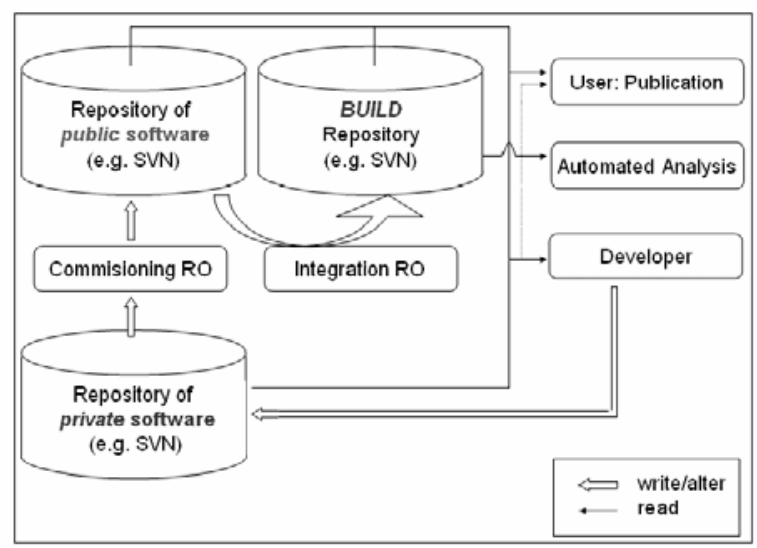

Fig.1 Software commissioning and integration.

are clear responsibilities documented in publicly available documents.

In Fig. 1 all changes in the public repository are to be accepted by the commissioning responsible officer. Formal criteria for acceptance cover exhaustive software documentations including application programming interface (API) description, references, user manual, and further application specific information describing the domain of each respective software component. In order to minimize the effort for the developer an automated documentation framework [3] is part of the concept. The framework generates a unified documentation on basis of in-code comments allowing an inclusion of any free configurable supplementary information.

In order to keep all the public software up-to-date, a special framework for a continuous build process is necessary. This is the domain of continuous software integration [4]. Continuous Integration uses a Build SVN repository to test the consistency of the whole software system. The process is supervised by an integration responsible officer (cf. Fig. 1). Currently, this workflow element is under assessment.

\section{Versioning}

At W7-X where software is developed by distributed groups of programmers it is inevitable to use a professional versioning system to manage all ongoing developments. The chosen open-source Subversion (SVN) has been replacing previously applied CVS software due to several benefits important for W7-X applications, cf. [5, 6] .

There exist repositories for several subject areas, one of them is the W7-X software repository. Providing a correct Kerberos (network authentication protocol [7]) password the current status of the W7-X repositories may be viewed via URL given in [2]. The current structure of the repository is shown in Table 1.

Table 1

SVN repositories for W7-X software.

\begin{tabular}{ll}
\hline Repository & Contents \\
\hline 3rdParty & $\begin{array}{l}\text { Software components developed outside } \\
\text { of IPP }\end{array}$ \\
data Analysis & $\begin{array}{l}\text { Statistical and neural networks codes, } \\
\text { information about data incertainties }\end{array}$ \\
dataSources & $\begin{array}{l}\text { Database software and definitions, e.g. } \\
\text { for the Magnetic Configuration } \\
\text { Database (MCDB) }\end{array}$ \\
gpurpos & $\begin{array}{l}\text { General purpose software, like } \\
\text { Programming Interface for Stellarator } \\
\text { Application (PISA) }\end{array}$ \\
theory & Theory codes \\
w7xc & Data and software for W7-X control \\
xdiag & $\begin{array}{l}\text { Diagnostic-specific software, as for } \\
\text { tomography }\end{array}$ \\
xdv & Domain of the data acquisition group \\
\hline
\end{tabular}

The Rechenzentrum Garching (computing centre which offers services for Max Planck institutes all over Germany) provides user authentication, secure access 
and firewall. SVN is accessible on three access levels: administrator (superuser), repository manager, and user identified with an IPP Kerberos password. No access to SVN is possible without a valid Kerberos password.

\section{Automated software documentation system}

\subsection{Motivation}

At W7-X software developments take place in form of new developments, required enhancement of existing software, implementation of external software packages, etc. On the other hand, there exist many heterogeneous groups, as experiment control, data acquisition, or theory departments, involved in both software development and application. Documentations of used software, if existing at all, are available in very different forms reaching from personal notes on a sheet of paper to detailed information accessible in internet. It turns out that many kinds of documentation can be automated. Therefore it seems to be possible to join existing software documentation tools and enhance the result to a uniform format, easy accessible for the users, preferably as a Web application. This would entail considerable advantages for the users

- $\quad$ Easy Web access (one URL to documentation of all W7-X software components)

- Unified documentation (same „look and feel“)

- $\quad$ All information (e.g. a reference) available with a simple mouse click

- Automated API documentation, using source code documentation tools

- $\quad$ Each single documentation can be individually supplemented with optional information

Automation of the API documentation forces the software developers to use a software versioning software and to proper structure and annotate their source codes (maybe with the aid of available prepared templates). The success of a unified documentation system is strongly based on the convenience of its application. To gain acceptance by the users this convenience is the leading non-functional requirement.

A thorough requirement analysis indicated that existing documentation frameworks do not meet all requirements, therefore a specific solution was developed.

\subsection{DocSys}

The automated documentation system for W7-X software was implemented as a prototype under the name of DocSys [3].

A functional diagram of DocSys is shown in Figure 2. According to defined metadata certain inputs are read and a documentation is created. A complete documentation contains some obligatory fields like To Do, User Manual, API Reference. At any time it is possible to add further project specific information. A click on the accordant field leads to more detailed information.

For an API documentation DocSys uses tools depending on the source programming language, Doxygen [8] for Java, C, C++, and F90doc [9] and Rdoc [10] for Fortran 90 and 95. A Cronjob provides a daily update of the source code documentation. If necessary API documentation tools can by triggered by hand at any time.

Like in case of SVN there are different access levels. An administrator sets general DocSys parameters (e.g. available versioning systems). A project manager defines metadata for his project (repositories for source codes, required computer environment, etc.; in the prototype version a total of 16 parameters are to be specified). And finally any user identified with an IPP Kerberos password can only view the documentation.

\subsection{Experiences with the DocSys prototype}

The feedback from the DocSys users has been predominant positive. Workload for the management is low. One project requires some 30 minutes for the setup, while the maintenance efforts are minimal.

DocSys version 1.0 is in preparation. This new release will be more comfortable to be used and managed, and offer some extended functionality as an interface to TRAC [11], search function for keywords, or sort by several parameters. 


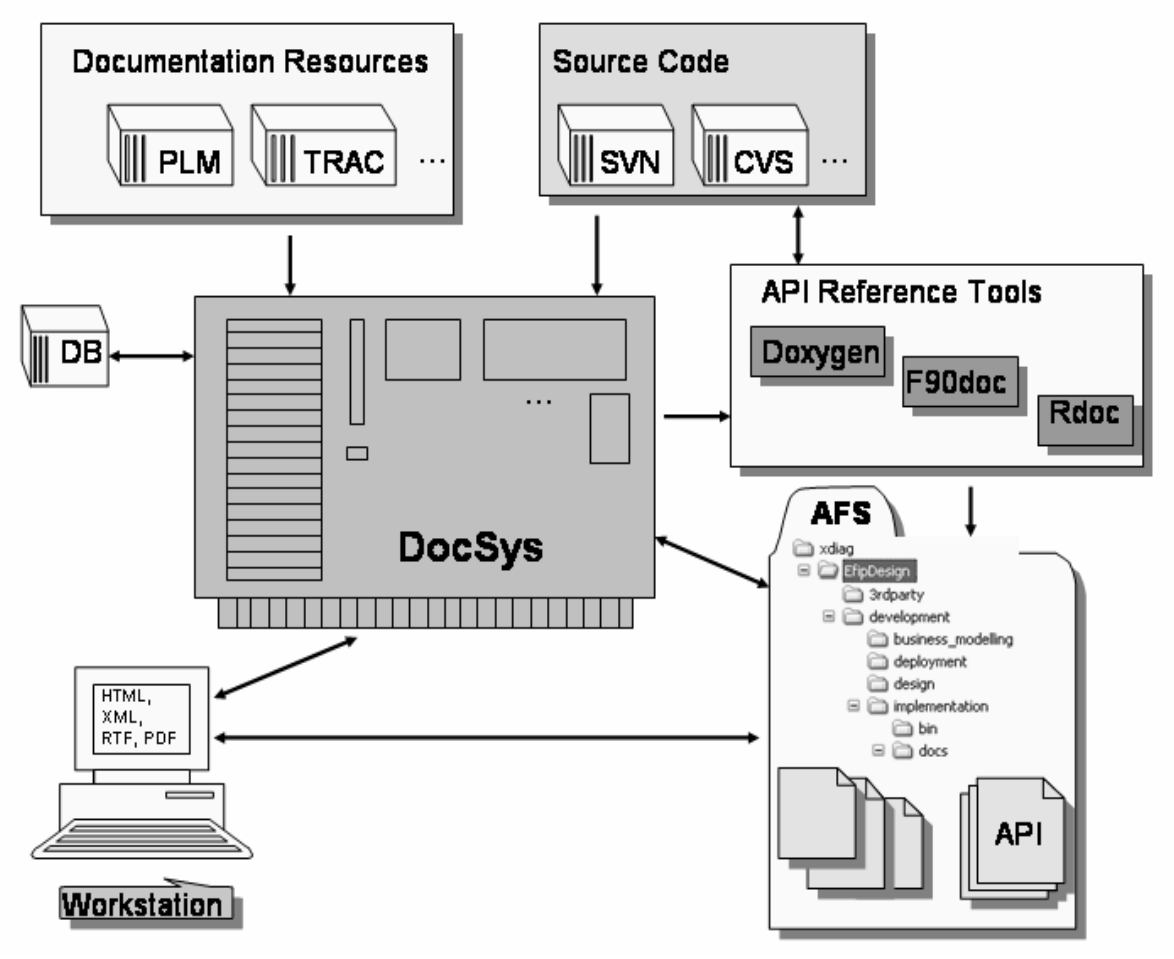

Fig. 2. DocSys functional diagram. PLM stands for product lifecycle management.

\section{Conclusion and Outlook}

A definition of the workflow elements for W7-X software developments is proceeding. Major requirements and actors were figured out and several measures for a workflow installation have been made. This covers software repositories and software documentation in addition to several directions documenting scope and responsibilities.

The hitherto gained experience with the implemented elements (SVN, software documentation tools, DocSys) meet the most users expectations and seem to be a good base for further extensions.

A completion of standards and the implementation of a software integration workflow are in progress.

\section{References}

[1] A. Werner, A. Dinklage, G. Kühner, H. Maaßberg, J. Schacht, J. Svensson, U.v. Toussaint, Yu. Turkin, Integrated Software Development for Wendelstein 7-X, 21st IAEA Fusion Energy Conference, Chengdu (2006)

[2] https://validatorwww.ipp-hgw.mpg.de/ (providing a valid IPP Kerberos password)

[3] A. Krüger, Ein automatisiertes Software Dokumentationssystem für Wendelstein 7-X (in German), Bachelor-Thesis, University of Applied Sciences Stralsund (2007)

[4] M. Fowler, Continuous Integration, http://www.martinfowler.com/ articles/ continuousIntegration.html/

[5] http://subversion.tigris.org/

[6] http://www.nongnu.org/cvs/

[7] http://tools.ietf.org/html/rfc4120

[8] http://www.stack.nl/ dimitri/doxygen/

[9] http://erikdemaine.org/software/f90doc/

[10] http://www.gfd-dennou.org/library/dcmodel/ rdoc-dennou/rdoc-f95/doc/files/README.html/

[11] http://trac.edgewall.org/ 